\title{
Minimally invasive right thoracotomy approach for mitral valve surgery in patients with previous sternotomy: A single institution experience with 173 patients
}

\author{
Michele Murzi, MD, Antonio Miceli, MD, PhD, Gioia Di Stefano, MD, Alfredo G. Cerillo, MD, \\ Pierandrea Farneti, MD, Marco Solinas, MD, and Mattia Glauber, MD
}

Objective: This study presents a review of our experience with minimally invasive mitral valve surgery (MIMVS) in patients with a previous cardiac procedure performed through a sternotomy over a 10-year period.

\begin{abstract}
Methods: From November 2003 to August 2013, 173 patients (age $61.3 \pm 12.4$ years) underwent reoperative MIMVS through a right minithoracotomy. Previous operations were coronary artery bypass grafting $(\mathrm{n}=49$; $28.6 \%)$, a mitral valve procedure $(\mathrm{n}=120 ; 70.1 \%)$, an aortic valve procedure $(\mathrm{n}=32 ; 18.7 \%)$, and other operations $(n=14 ; 8.1 \%)$. The mean euroSCORE was $11.2 \pm 3.8$. The time to redo surgery was $6.9 \pm 4.2$ years.

Results: Procedures were performed with central aortic cannulation in 55 patients $(31.7 \%)$ and peripheral cannulation in $118(68.3 \%)$. A transthoracic clamp was used in 58 patients $(33.5 \%)$, an endoaortic balloon in $72(41.6 \%)$, hypothermic ventricular fibrillation in $23(13.2 \%)$, and beating heart in $20(11.5 \%)$. Mean cardiopulmonary bypass and crossclamp times were $160 \pm 58$ minutes and $82 \pm 49$ minutes, respectively. Mitral repair was performed in 53 patients (30.6\%). Forty-three patients $(24.7 \%)$ had an additional cardiac procedure. Conversion to sternotomy was necessary in 2 patients $(1.1 \%)$ and reoperation for bleeding in 11 patients $(6.3 \%)$. Thirty-day mortality was $4.1 \%(\mathrm{n}=7)$. Major morbidities included stroke $(\mathrm{n}=11 ; 6 \%)$ and new-onset dialysis requirement $(\mathrm{n}=4 ; 2.3 \%)$. The mean blood transfusion requirement was $1.4 \pm 1.1$ units. Mean follow-up was $3.3 \pm 2.6$ years. Survival at 1,5 , and 10 years was $93.1 \% \pm 1.9 \%, 87.5 \% \pm 2.7 \%$, and $79.7 \% \pm$ $3.8 \%$, respectively.
\end{abstract}

Conclusions: Reoperative mitral valve surgery can be safely performed through a right minithoracotomy with good early and late outcomes. The avoidance of extensive surgical dissection, optimal valve exposure, and low blood transfusion are the main advantages of this technique. (J Thorac Cardiovasc Surg 2014;148:2763-8)

In recent years, less invasive procedures have emerged as the new trend in mitral valve surgery. Since the first report by Carpentier and colleagues, ${ }^{1}$ the conviction that minimally invasive mitral valve surgery (MIMVS) could be performed with similar hospital mortality to the standard approach, but with less blood transfusion, earlier recovery of daily activities, and higher patient satisfaction, have driven its diffusion in most centers worldwide. Although a large volume of literature on primary MIMVS is available, only a few studies have been reported on the use of this technique in patients who have undergone a previous cardiac surgical procedure. ${ }^{2}$ In 2003, we started an MIMVS program and from the beginning, video-assisted right thoracotomy approach has been the approach of choice for reoperative mitral valve surgery. The aim of the present

\footnotetext{
From the Fondazione Toscana Gabriele Monasterio, G. Pasquinucci Heart Hospital, Massa, Italy.

Disclosures: Authors have nothing to disclose with regard to commercial support. Received for publication Feb 25, 2014; revisions received July 26, 2014; accepted for publication July 29, 2014; available ahead of print Sept 12, 2014.

Address for reprints: Michele Murzi, MD, G. Pasquinucci Heart Hospital, Via Aurelia Sud, 100, 54100 Massa, Italy (E-mail: michelem@ftgm.it). $0022-5223 / \$ 36.00$

Copyright (c) 2014 by The American Association for Thoracic Surgery http://dx.doi.org/10.1016/j.jtcvs.2014.07.108
}

study is therefore to review our experience with MIMVS in patients with a previous cardiac operation performed through a median sternotomy and to assess the effectiveness and the safety of this approach.

\section{PATIENTS AND METHODS \\ Patient Selection and Data Collection}

This was a retrospective, observational, cohort study of prospectively collected data from consecutive patients who underwent reoperative MIMVS via a right minithoracotomy at the G. Pasquinucci Heart Hospital between November 2003 and August 2013. The data collection form is entered in an institutional database and includes 5 sections that are filled in consecutively by anesthetists, surgeons, and nurses in the intensive care unit, high-dependency unit, and wards. The resulting base sample contained detailed clinical information on 173 patients. No patients were excluded. The study was approved by the clinical audit committee of the G. Pasquinucci Heart Hospital to meet ethical and legal requirements, and individual consent was waived.

Follow-up was conducted from September to October 2013 by telephone interview using a structured questionnaire and was $100 \%$ complete.

At our institution, right minithoracotomy is considered the preferred approach in patients with a previous sternotomy, who require a redo mitral valve operation alone or with other cardiac procedures such as tricuspid valve surgery, atrial fibrillation ablation, and closure of an atrial septal defect. Contraindications for this approach are: previous right pleural disease, previous right thoracotomy, and the need for a concomitant procedure such as coronary artery bypass grafting, aortic valve replacement, ascending aorta replacement, or left ventricular reshaping. 


$$
\begin{aligned}
& \text { Abbreviations and Acronyms } \\
& \begin{aligned}
\text { CI } & =\text { confidence interval } \\
\text { HR } & =\text { hazard ratio } \\
\text { MIMVS } & =\text { minimally invasive mitral valve surgery } \\
\text { OR } & =\text { odds ratio }
\end{aligned}
\end{aligned}
$$

\section{Definitions}

Hospital mortality included all deaths within 30 days of operation irrespective of where the death occurred and all deaths in hospital after 30 days among patients who had not been discharged after the index operation. Our definition of perioperative stroke included any new temporary or permanent, focal or global neurologic deficit. Perioperative stroke was diagnosed during clinical assessment by physicians involved in the daily care of patients, and was confirmed by computed tomography or magnetic resonance imaging whenever possible. The diagnosis of stroke was also confirmed and documented by staff neurologists. Renal complications included acute renal failure, defined as the requirement for hemodialysis or an increased creatinine level ( $>200 \mathrm{mmol} / \mathrm{L})$. A diagnosis of postoperative myocardial infarction was based on the presence of new $\mathrm{Q}$ waves greater than 0.04 milliseconds and/or a reduction in $\mathrm{R}$ waves greater than $25 \%$ in at least 2 contiguous leads on an electrocardiogram. Pulmonary complications included chest infection, ventilation failure, reintubation, and tracheostomy.

\section{Surgical Technique}

Our operative technique has been described previously. ${ }^{3}$ In brief, it consists of a small $(5-7 \mathrm{~cm})$ right anterolateral thoracotomy in the third or fourth intercostal space. After incision, a soft tissue retractor is inserted and the intercostal space is gently spread with a retractor without rib cutting. Two trocars are inserted in the thorax to allow positioning of a ventricular vent, $\mathrm{CO}_{2}$ insufflator, and a camera device. Based on our belief that antegrade perfusion has advantages over retrograde flow, ${ }^{4,5}$ we adopt central cannulation whenever possible. Under direct visualization, the aorta is exposed up to the origin of the innominate artery after which 2 concentric purse string sutures for direct arterial cannulation are placed in a standard fashion into the ascending aorta. The cannulation site is generally chosen by identifying the transverse sinus as the orientation where the aortic crossclamp will be placed. It is sufficient if the cannulation site stays at some distance above the crossclamp level. Direct ascending aorta cannulation is performed under direct vision. The lungs must be deflated before aortic cannulation. The adventitia is prepared with scissors and the aortic cannula is advanced into the aorta. ${ }^{6}$ Femoral artery cannulation is used in those patients who have an obvious contraindication to ascending aorta cannulation such as patent saphenous vein grafts, previous ascending aorta replacement, and/or severe adhesions around the ascending aorta. Venous drainage is achieved by a long venous cannula introduced percutaneously into the femoral vein and positioned under transesophageal echo guidance in the right atrium with the tip in the superior vena cava. In the first 5 patients requiring an additional procedure in the right atrium, an adjunctive right internal jugular vein cannula was inserted preoperatively by the anesthetist. However, in the last 28 patients who required opening of the right atrium, we used a single 2-stage femoral venous cannula (RAP Venous Cannula, Estech Inc, San Ramon, Calif), which allows the superior and inferior venae cavae to drain simultaneously. ${ }^{6}$ To achieve good myocardial protection, if the aorta can be safely dissected and clamped, we prefer to clamp the ascending aorta directly with a flexible transthoracic clamp and to deliver antegrade cold crystalloid cardioplegia. Endoaortic balloon clamping with antegrade cardioplegia is used in patients in whom the ascending aorta cannot be safely mobilized. Hypothermic ventricular fibrillation $\left(28^{\circ} \mathrm{C}-30^{\circ} \mathrm{C}\right.$ core temperature) or beating heart surgery is used in patients with patent coronary artery bypass grafts or severe ascending aortic calcifications.

The mitral valve is exposed through a standard left paraseptal atriotomy with the aid of an atrial retractor supported by an external mechanical arm inserted in the thorax through a right parasternal hole. Mitral repair is performed using the standard Carpentier technique with a combination of thoracoscopic assistance and direct vision. Surgical ablation of the atrial fibrillation is performed with standard endocardial left atrial lesions using a monopolar radiofrequency probe concomitant with left atrial appendage exclusion.

Deairing the heart is achieved using a 2-stage procedure. First, the table is lifted head up, the heart is filled, both lungs are ventilated, and air is removed from the left atrium with a small forceps inserted through the suture line. The bed is then lifted head down, the aortic clamp is released, and air is removed via the transmitral vent and the aortic vent. Our favored operative setup is shown in Figure 1.

\section{Statistical Analyses}

Categorical variables are expressed as proportions and continuous variables as means \pm standard deviation. Stepwise multivariable regression analysis was used to identify independent risk factors for 30-day mortality, with a significance level of .05 used for both entry and selection. Similarly, multivariable Cox proportional hazard modeling was used to identify independent risk factors for all-cause mortality. Covariates under consideration for all models were all baseline characteristics (as listed in Table 1). Overall survival was estimated using the Kaplan-Meier method and was expressed as a percentage. All reported $P$ values are 2 -sided. Statistical analysis was performed with SPSS version 19.0 (SPSS, Inc, Chicago, Ill).

\section{RESULTS}

\section{Patient Characteristics and Operative Data}

Preoperative characteristics of the patients are given in Table 1 and their previous cardiac operations in Table 2. Mean time to redo surgery was $6.9 \pm 4.2$ years. This was the third intervention in 22 patients, the fourth in 4 patients, and the seventh in 1. Mean preoperative euroSCORE (European System for Cardiac Operative Risk Evaluation) was $11.2 \pm 3.8$. The operation was performed with direct ascending aorta cannulation in 55 patients $(31.7 \%)$ and femoral artery cannulation in $118(68.3 \%)$. Myocardial protection was achieved with aortic clamping (transthoracic crossclamp in 58 patients and endoaortic balloon occluder in 72) and cold crystalloid cardioplegia. The remaining patients underwent surgery during hypothermic ventricular fibrillation $(\mathrm{n}=23 ; 13.2 \%)$ or beating heart $(\mathrm{n}=20 ; 11.5 \%)$. The distribution of valvular procedures performed and concomitant operative procedures is presented in Table 3. Mean cardiopulmonary bypass and aortic crossclamp times were $160 \pm 48$ minutes and 82 \pm 49 minutes, respectively. Conversion to sternotomy was required in 2 patients during the procedure: due to atrioventricular disruption in 1 patient and extensive adhesions on the chest wall in the other. A mitral repair was performed in 53 patients $(30.6 \%)$. In patients without a previous mitral procedure, the rate of repair was $82 \%$ and $76 \%$ in the subgroup of patients with a degenerative disease. 


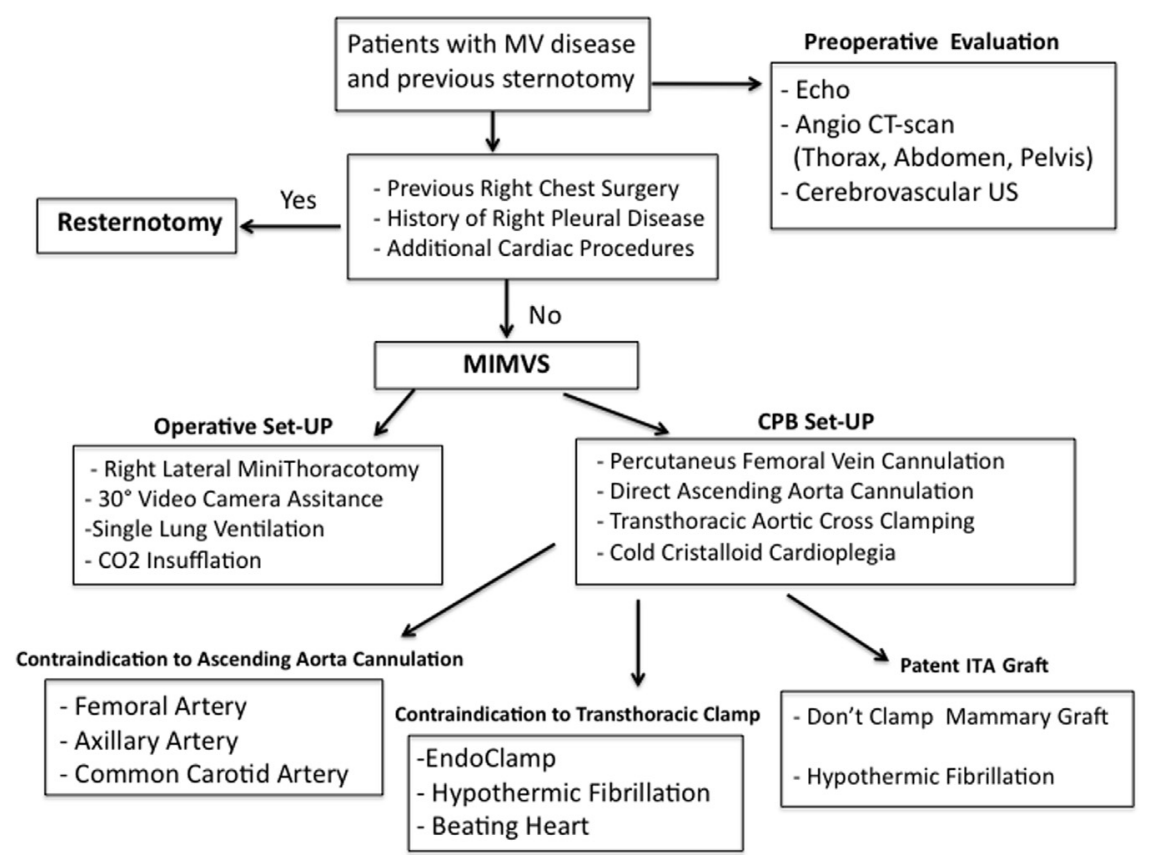

FIGURE 1. Diagram of our favored operative setup. $M V$, Mitral valve; $C T$, computed tomography; $U S$, ultrasonography; MIMVS, minimally invasive mitral valve surgery; $C P B$, cardiopulmonary bypass; ITA, internal thoracic artery.

\section{Postoperative Data}

The 30-day mortality rate was $4.1 \%(n=7)$. Causes of death were low cardiac output syndrome in 3 patients, multiorgan failure in 1 , neurologic complication in 1 , and sepsis in 1 . Stroke was observed in 11 patients $(6.3 \%)$, transient in 9 patients and permanent in 2. Although not significant, we observed a trend suggesting a higher rate of stroke in patients who underwent the operation with femoral artery cannulation. Postoperative acute renal failure occurred in $11.5 \%(\mathrm{n}=20)$. Hemodialysis was necessary in 4 of these patients $(2.3 \%)$. Pulmonary complications were observed in 20 patients $(11.5 \%)$. No perioperative myocardial infarction was observed. Reoperations for bleeding were necessary in 11 patients $(6.3 \%)$. Mean time of postoperative mechanical ventilatory support was $14 \pm 18$ hours. Mean length of stay in the intensive care unit was $2.1 \pm 4.3$ days and mean hospital stay was $10 \pm 5.9$ days. A morbidity analysis for all patients is shown in Table 4. Early reoperation ( $<30$ days) was necessary in 1 patient because of valve repair failure. The operation was reperformed through the right minithoracotomy and the valve was rerepaired. Blood transfusions were required in 68 patients $(40.7 \%)$ at a mean $1.4 \pm 1.1$ units/patient. Echocardiography at hospital discharge revealed the absence or minimal residual mitral regurgitation in all patients who underwent mitral valve repair except 1 patient who had a moderate mitral regurgitation. Independent risk predictors of 30-day mortality were endocarditis (odds ratio [OR], 5.84; 95\% confidence interval $[\mathrm{CI}], 1.09-31.1 ; P=.03$ ) and peripheral arteriopathy (OR, 6.26; 95\% CI, 1.29-30.1; $P=.02$ ).

\section{Follow-up}

Mean follow-up was $3.3 \pm 2.6$ years and was $100 \%$ complete. Late death occurred in 17 patients ( 1 sudden death, 11 cardiac-related deaths, and 5 non-cardiac-related deaths). The mean New York Heart Association class at follow-up was $1.5 \pm 0.6$. There was 1 late reintervention at 1.1 year for mitral prosthesis endocarditis. Survival at 1, 5 months, and 10 years was $93.1 \% \pm 1.9 \%, 87.5 \% \pm 2.7 \%$, and $79.7 \% \pm 3.8 \%$, respectively (Figure 2). Echocardiographic follow-up of the 53 patients who underwent mitral repair revealed moderate mitral regurgitation in 7 patients. All the others had none or trivial mitral regurgitation.

In the multivariate Cox proportional hazards model, the variables that emerged as independent predictors of longterm mortality were age (hazard ratio [HR], 1.06; 95\% CI, 1.02-1.21; $P=.04$ ) and endocarditis (HR, 3.31; 95\% CI, $0.98-11.1 ; P=.03)$.

\section{COMMENT}

At our center, right minithoracotomy with ascending aorta cannulation, femoral venous drainage, and transthoracic aortic clamping have become the approach of choice for mitral valve operations since $2003,{ }^{7}$ Early in our experience, we recognized the advantages of this technique for reoperative procedures. With this access, the heart, great vessels, and patent bypass grafts are far away from the working field and were not injured in any of our patients. Avoidance of previously dissected tissue and less need for surgical dissection can prevent the need for transfusion due to postoperative hemorrhage and the need for reexploration due 
TABLE 1. Preoperative characteristics

\begin{tabular}{lcc}
\hline & Number $(\mathbf{N}=\mathbf{1 7 3})$ & $\%$ \\
\hline Age, y \pm SD & $61.3 \pm 12.4$ & \\
Age 80 y & 14 & 8.1 \\
Female & 80 & 46.7 \\
Smoker & 57 & 33.3 \\
Extracardiac arteriopathy & 33 & 19.3 \\
Hypertension & 100 & 58.4 \\
Diabetes & 27 & 15.7 \\
COPD & 20 & 11.6 \\
Chronic renal failure & 8 & 4.6 \\
History of atrial fibrillation & 89 & 52 \\
Cerebrovascular disease & 26 & 15.2 \\
NYHA functional class III-IV & 94 & 54.9 \\
LVEF (\%) & $50 \pm 16$ & \\
LVEF <35\% & 27 & 15.7 \\
Predominant valve lesion & & \\
MV regurgitation & 153 & 89.4 \\
MV stenosis & 20 & 10.6 \\
TV regurgitation & 47 & 26.1 \\
Cause & & \\
Functional & 16 & 1.7 \\
Ischemic & 24 & 18.7 \\
Rheumatic & 32 & \\
Endocarditis & 16 & \\
Degenerative & 53 & \\
Congenital & 36 & \\
Prosthesis dysfunction & 26 & \\
\hline
\end{tabular}

$S D$, Standard deviation; $C O P D$, chronic obstructive pulmonary disease; $N Y H A$, New York Heart Association; $L V E F$, left ventricular ejection fraction; $M V$, mitral valve; $T V$, tricuspid valve.

to bleeding. ${ }^{2,8-13}$ In our series, a transfusion was required in $40.7 \%$ of the patients and the transfusion was required at a mean of $1.4 \pm 1.1$ units/patient. Concerns about MIMVS are related to suboptimal exposure and prolonged cardiopulmonary bypass time. ${ }^{14}$ In our experience, right minithoracotomy allows straight access to the mitral valve without the need for extensive cardiac mobilization as in standard redo procedures. This is supported by the fact that in our series, no patient required conversion to sternotomy because of difficult mitral valve exposure or major vascular injury. The minimally invasive approach guarantees a safe and excellent view of all the valve apparatus, allowing all types of repair techniques such as neochordal placement and anterior leaflet repair. In our series, mitral valve repair was performed in $82 \%$ of patients without previous mitral valve surgery and in $25 \%$ of patients with a

TABLE 2. Previous operation

\begin{tabular}{lcc}
\hline \multicolumn{1}{c}{ Type } & Number & $\%$ \\
\hline Mitral procedure & 120 & 70.1 \\
CABG & 49 & 28.6 \\
AVR & 32 & 18.7 \\
Aorta procedure & 3 & 1.7 \\
Tricuspid procedure & 11 & 6.4 \\
\hline
\end{tabular}

$C A B G$, Coronary artery bypass grafting; $A V R$, aortic valve replacement.
TABLE 3. Operative data

\begin{tabular}{lcc}
\hline & Number & $\%$ \\
\hline Femoral venous cannulation & 171 & 100 \\
Jugular vein cannulation & 5 & 2.9 \\
Femoral artery cannulation & 118 & 68.3 \\
Ascending aortic cannulation & 55 & 31.7 \\
Direct aortic clamping & 58 & 33.5 \\
Endoaortic balloon clamping & 72 & 41.6 \\
Hypothermic ventricular fibrillation & 23 & 13.2 \\
Beating heart & 20 & 11.5 \\
CPB time, min \pm SD & $160 \pm 58$ & \\
Crossclamp time, min \pm SD & $82 \pm 49$ & \\
Conversion to sternotomy & 2 & 1.1 \\
Procedures & & \\
Mitral valve repair & 53 & 30.6 \\
$\quad$ Annuloplasty & 53 & 100 \\
$\quad$ Leaflet procedure & 32 & 65 \\
$\quad$ Neochordae & 9 & 18.3 \\
Mitral valve replacement & 114 & 65.8 \\
$\quad$ Bioprosthesis & 40 & 35 \\
$\quad$ Mechanical prosthesis & 73 & 65 \\
Paraprosthetic leak repair & 6 & 3.4 \\
Tricuspid valve repair & 33 & 19.1 \\
AF ablation & 5 & 2.8 \\
ASD/PFO closure & 5 & 2.8 \\
\hline$C P B$, Cardiopulmonary bypass; $S D$, standard deviation; $A F$, atrial fibrillation; & $A S D$, \\
atrial septal defect; $P F O$, patent foramen ovale. & &
\end{tabular}

previous mitral valve repair. The right chest approach did not impede the replacement of a mitral prosthesis or repair of a paravalvular leak. In our series, we have also applied the technique to patients with active endocarditis. However, in this subset of patients, it is extremely important to confirm preoperatively the absence of any trigone or aortic valve involvement. With regard to cardiopulmonary bypass time, we found that our operative results compare favorably with data reported in other series on mitral valve reoperation performed through a similar approach as well as through resternotomy, ${ }^{8-13,15,16}$ These results are encouraging considering that adjunctive procedures were associated with mitral valve surgery in about $25 \%$ of our patients.

Reoperative MIMVS has been shown to have similar mortality and morbidity compared with standard sternotomy procedures. ${ }^{2,8,15}$ However, some studies have reported a greater incidence of neurologic complications with a minimally invasive approach. ${ }^{17-20}$ Svensson and colleagues ${ }^{17}$ showed that right thoracotomy carries an unacceptable risk of stroke compared with resternotomy during reoperative mitral valve surgery (right thoracotomy $7.5 \%$ vs $2.7 \%$ resternotomy; $P=.006$ ). The investigators identified the use of retrograde arterial cannulation, ventricular fibrillatory arrest, and incomplete deairing as risk factors for the increased risk of stroke. In response to this study, in 2010 Crooke and colleagues ${ }^{4}$ analyzed a series of 905 patients who underwent reoperative mitral valve procedures 
TABLE 4. Postoperative morbidity

\begin{tabular}{lcc}
\hline \multicolumn{1}{c}{ Complication } & Number & $\%$ \\
\hline 30-d mortality & 7 & 4.1 \\
Stroke & 11 & 6.3 \\
$\quad$ Transient & 9 & 81.8 \\
$\quad$ Permanent & 2 & 18.2 \\
Delirium & 20 & 11.5 \\
Postoperative AMI & 0 & 0 \\
Pulmonary complications & 20 & 11.5 \\
Renal failure & 20 & 11.5 \\
New-onset dialysis & 4 & 2.3 \\
Reoperation for bleeding & 11 & 6.3 \\
Atrioventricular block & 6 & 3.4 \\
Postoperative AF & 54 & 31.2 \\
\hline
\end{tabular}

$A M I$, Acute myocardial infarction; $A F$, atrial fibrillation.

through a median sternotomy $(612 ; 67.6 \%)$, right anterior thoracotomy $(242 ; 26.7 \%)$, and left posterior thoracotomy in $(51 ; 5.6 \%)$. In their analysis, the incidence of stroke was linked to retrograde arterial perfusion and not to the location of the incision, suggesting a more liberal use of antegrade perfusion. Recently, we carried out a similar analysis on 1280 nonreoperative MIMVS and our results confirm the negative impact of retrograde arterial cannulation on neurologic outcome. ${ }^{21}$ Because of concerns regarding the negative impact of retrograde perfusion on neurologic outcome, our strategy has progressively evolved from femoral artery cannulation to direct ascending aorta cannulation. In practice, we avoid retrograde perfusion whenever possible, restricting femoral artery cannulation only to circumstances where direct ascending aorta cannulation is prohibitive, such as patent coronary bypass or hostile mediastinum. In addition, as part of our stroke prevention protocol, we always use $\mathrm{CO}_{2}$ insufflation in the surgical field to reduce the amount of intracardiac air, and we leave the transmitral and aortic root vent in place until the heart is fully beating and no more bubbles are seen on transesophageal echocardiography. Since adopting this procedure, we have noted a significant reduction in stroke rate, from $8.3 \%$ in the first 123 patients to $2 \%$ in the last 50 patients.

Reoperative MIMVS in the presence of patent left internal thoracic grafts represents a particular challenge because these grafts cannot be isolated and clamped during cardioplegia delivery. In this subset of patients, we have routinely used systemic cooling and ventricular fibrillation. The absence of perioperative myocardial infarction and the low incidence of low cardiac output syndrome are evidence of the success of this strategy. Our results reconfirm the safety and efficacy of not clamping a patent left internal thoracic artery graft and mitigate its presence by the strategy of additional core cooling. ${ }^{22}$ Alternatively, in this subset of patients, systemic cooling with cardiac arrest by administration of cold antegrade crystalloid cardioplegia or beating heart surgery may be safely performed. ${ }^{23}$

In our series, the 30 -day mortality $(4.1 \%)$ compared favorably with other studies in which mitral valve reoperations were performed either through a right thoracotomy ${ }^{8-13}$ or through a standard sternotomy. ${ }^{15,16}$ Although it is difficult to compare results from different series because of differences in patient populations, we can conclude that our observed mortality rate of $4.1 \%$ confirms that MIMVS

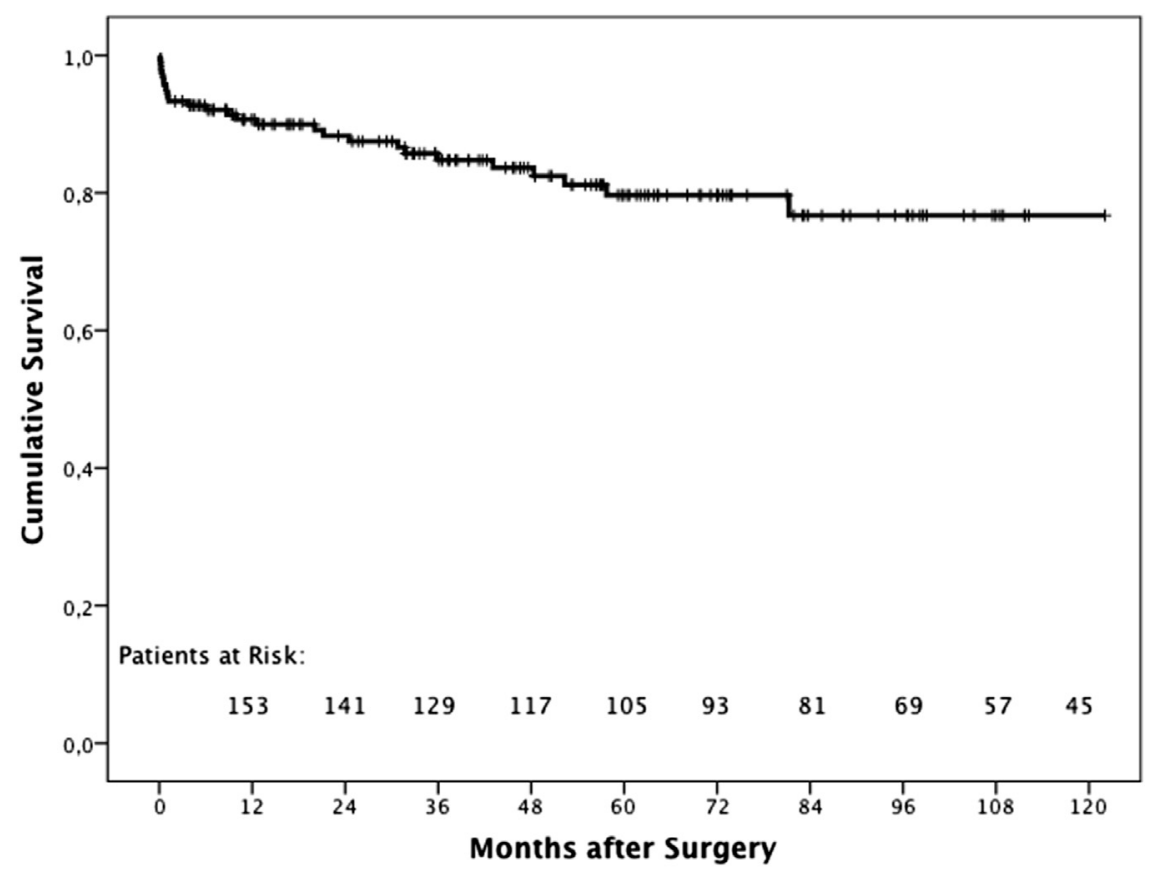

FIGURE 2. Kaplan-Meier survival curve. 
may represent a valid alternative to standard sternotomy mitral valve reoperation. Of particular interest is the fact that mortality in our study was significantly lower than the expected mortality predicted by the euroSCORE (observed/expected ratio, 0.12). Although it is widely accepted that the euroSCORE tends to overestimate the operative mortality of patients undergoing valvular surgery, ${ }^{24}$ we believe that the practical and potential benefits of the minithoracotomy approach, and in particular reduced surgical dissection, good exposure, good myocardial protection, and low need for blood transfusion, might have contributed to these results.

Additional procedures, such as tricuspid valve surgery or atrial fibrillation ablation, can be safely performed through a right minithoracotomy. At the beginning of our experience, right atrial procedures were performed with bicaval cannulation and tapes around the venae cavae. However, in redo procedures, placement of caval snares may be particularly challenging through a very small incision and may expose the patient to the risks of caval bleeding and laceration. Therefore, since April 2007, we have shifted to a double-stage single femoral venous cannula that has 2 distal perforated sections separated by a nonperforated segment of $15 \mathrm{~cm}$ in length, ideated to drain the inferior and superior venae cavae simultaneously. The use of this cannula offers many advantages over bicaval cannulation. First, it avoids complications related to the insertion of a right internal jugular vein cannula, such as bleeding, hematoma, inadvertent carotid lesions, and pneumothorax. Second, this cannula achieves a satisfactory bloodless working field without occluding the venae cavae. ${ }^{6}$

\section{Limitations of the Study}

There are several limitations of this study. This is a retrospective study at a single center and thus it is inevitably biased in patient selection and the technique used. In addition, our report includes our initial experience. The fact that we cannot compare our series with a cohort of patients undergoing resternotomy is another important limitation.

\section{CONCLUSIONS}

Our study suggests that reoperative mitral valve surgery can be safely performed through a right minithoracotomy with low morbidity and mortality and with acceptable midterm results. The avoidance of extensive surgical dissection, good exposure of the valve, low requirement for blood transfusion, and short hospital stay are the main advantages of this technique.

\section{References}

1. Carpentier A, Loulmet D, Aupecle B, Berrebi A, Relland J. Computer-assisted cardiac surgery. Lancet. 1999;353:379-80.

2. Murzi M, Solinas M, Glauber M. Is a minimally invasive approach for reoperative mitral valve surgery superior to standard resternotomy? Interact Cardiovasc Thorac Surg. 2009;9:327-32.
3. Glauber M, Karimov J, Farneti PA, Cerillo AG, Santarelli F, Ferrarini M, et al. Minimally invasive mitral valve surgery via right minithoracotomy. Multimed Man Cardithorac Surg. 2009;2009. mmcts.2008.003350.

4. Crooke GA, Schwartz FC, Ribakove GH, Ursomanno P, Gogoladze G, Culliford AT, et al. Retrograde arterial perfusion, not incision location, significantly increases the risk of stroke in reoperative mitral valve procedures. Ann Thorac Surg. 2010;89:723-30.

5. Grossi EA, Loulmet DF, Schwartz CF, Solomon B, Dellis SL, Culliford AT, et al. Minimally invasive valve surgery with antegrade perfusion strategy is not associated with increased neurologic complications. Ann Thorac Surg. 2011;92: 1346-50.

6. Glauber M, Murzi M, Solinas M. Central aortic cannulation for minimally invasive mitral valve surgery through right minithoracotomy. Ann Cardiothorac Surg. 2013;2:839-40.

7. Murzi M, Kallushi E, Solinas M, Glauber M. Video-assisted right atrial surgery with a single two stage femoral venous cannula. Interact Cardiovasc Thorac Surg. 2009;9:9-10.

8. Murzi M, Cerillo AG, Bevilacqua S, Gasbarri T, Kallushi E, Farneti P, et al. Enhancing departmental quality control in minimally invasive mitral valve surgery: a single-institution experience. Eur J Cardiothorac Surg. 2012;42: 500-6.

9. Botta L, Cannata A, Bruschi G, Fratto P, Taglieri C, Russo CF, et al. Minimally invasive approach for redo mitral valve surgery. J Thorac Dis. 2012;5: S686-93.

10. Modi P, Hassan A, Chitwood WR. Minimally invasive mitral valve surgery: a systematic review and meta-analysis. Eur J Cardiothorac Surg. 2008;34: 943-52.

11. Casselman F, La Meir M, Jeanmart H, Mazzarro E, Coddens J, Van Praet F, et al. Endoscopic mitral and tricuspid valve surgery after previous cardiac surgery. Circulation. 2007;116:270-5.

12. Seeburger J, Borger MA, Falk V, Passage J, Walther T, Doll N, et al. Minimally invasive mitral valve surgery after previous sternotomy: experience in 181 patients. Ann Thorac Surg. 2009;87:709-14.

13. Meyer SR, Szeto WY, Augoustides JGT, Morris RJ, Vernick WJ, Paschal D, et al. Reoperative mitral valve surgery by the port access minithoracotomy approach is safe and effective. Ann Thorac Surg. 2009;87:1426-30.

14. Arcidi JM, Rodriguez E, Elbeery JR, Nifong LW, Efird JT, Chitwood RW. Fifteen-year experience with minimally invasive approach for reoperations involving the mitral valve. J Thorac Cardiovasc Surg. 2012;143:1062-8.

15. Baldwin JC. Editorial (con) re minimally invasive port-access mitral valve surgery. J Thorac Cardiovasc Surg. 1998;115:563-4.

16. Burfeind WR, Glower DD, Davis RD, Landolfo KP, Lowe JE, Wolfe WG. Mitral surgery after prior cardiac operation: port-access versus sternotomy or thoracotomy. Ann Thorac Surg. 2002;74:1323-5.

17. Borger MA, Yau TM, Rao V, Scully HE, David TE. Reoperative mitral valve replacement: importance of preservation of the subvalvular apparatus. Ann Thorac Surg. 2002;74:1482-7.

18. Svensson LG, Gillinov AM, Blackstone EH, Houghtaling PL, Kim KH, Pettersson GB, et al. Does right thoracotomy increase the risk of mitral valve reoperation? J Thorac Cardiovasc Surg. 2007;134:677-82.

19. Gammie JS, Zhao Y, Peterson ED, O’Brien SM, Rankin JS, Griffith BP. Lessinvasive mitral valve operations: trends and outcomes from the Society of Thoracic Surgeons Adult Cardiac Surgery Database. Ann Thorac Surg. 2010; 90:1401-8.

20. Ricci D, Pellegrini C, Aiello M, Alloni A, Cattadori B, D'Armini AM, et al. Portaccess surgery as elective approach for mitral valve operation in re-do procedures. Eur J Cardiothorac Surg. 2010;37:920-5.

21. Murzi M, Cerillo AG, Miceli A, Bevilacqua S, Kallushi E, Farneti P, et al. Antegrade and retrograde arterial perfusion strategy in minimally invasive mitralvalve surgery: a propensity score analysis on 1280 patients. Eur J Cardiothorac Surg. 2013;43:167-72.

22. Smith RL, Ellman PI, Thompson PW, Girotti ME, Mettler BA, Ailawadi G, et al. Do you need to clamp a patent left internal thoracic artery-left anterior descending graft in reoperative cardiac surgery? Ann Thorac Surg. 2009;87: 742-7.

23. Thompson MJ, Behranwala A, Campanella C, Walker WS, Cameron EW. Immediate and long-term results of mitral prosthetic replacement using a right thoracotomy beating heart technique. Eur J Cardiothorac Surg. 2003;24:47-51.

24. Bhatti F, Grayson AD, Grotte G, Fabri BM, Au J, Jones M, et al. The Logistic EUROSCORE in cardiac surgery: how well does it predict operative risk? Heart. 2006;92:1817-20. 